\title{
A NEW RELATION BETWEEN PREFERRED INCLINATIONS AND MEAN RADIAL VELOCITY OF THE CLUSTER
}

\author{
B. Aryal* and D. Yadav* \\ *Central Department of Physics, Tribhuvan University, Kirtipur, Nepal.
}

\begin{abstract}
We worked on POSSII and ESO films and measured the diameters and position angles of 5,688 galaxies in 34 clusters using 25-fold magnification microscope. The inclinations of individual galaxies are determined using Holmberg's method and their distributions are studied. The expected distributions are compared with the fitted curves. For this, we used linear, polynomial (second order) and Gaussian fits. We noticed a relation between the preferred inclination angle of the cluster and their mean radial velocity. Possible explanations of the result will be discussed.
\end{abstract}

Keywords: Galaxies; Evolution - galaxies; Formation - galaxies; Statistics - galaxies; Clusters.

\section{INTRODUCTION}

The galaxy inclination measures the angle at which a spiral galaxy is tilted from us. A galaxy with a $90^{\circ}$ galaxy inclination is seen edge on. One with a $0^{\circ}$ galaxy inclination is seen face on. The inclination ( $i$ ) is defined as the angle between the spin vector of a galaxy and the line-of-sight. If $i<0^{\circ}$ an ellipse with the major ( $a$ ) and minor axes $(b)$ instead of a circle is projected onto the sphere for which both axes can be measured. The method for determination of the inclination is based on two assumptions: (1) the galaxy is circular-like if seen face-on and (2) the distance to it is large enough to have no perspective distortions. This angle can be estimated with Holmberg's (1946) formula ${ }^{1}$. The greater the ellipticity the more the galaxy's disk inclined with respect to our lineof-sight. In practice, we measure the ellipticity by examining the contours of equal surface brightness (isophotes) in an image.

The Second Palomar Observatory Sky Survey (POSS-II) was performed during 1980-1991 that made use of better, faster films and an upgraded telescope. The observatory currently consists of three large telescopes: the 200 inch (5.1 m) Hale telescope, the 48 inch (1.2 m) Samuel Oschin telescope, and a 60 inch (1.5 m) reflecting telescope ${ }^{2}$. The Oschin Schmidt was given an achromatic corrector and provisions for autoguiding. Images were recorded in three wavelengths: blue (IIIaJ), red (IIIaF) and near infrared (IVN) plates, respectively ${ }^{3}$. Until the completion of the Two Micron All Sky Survey (2MASS) and Solon Dizitised Sky Survey (SDSS) POSS-II was the most extensive wide-field sky survey ever.

The preferred inclinations of galaxies in clusters and their relations to the cluster redshift might be important in order to understand evolution of galaxies in the cluster ${ }^{4,5,6}$. We present an analysis of the inclinations of 5,688 spiral galaxies in 35 clusters and discuss their relations to the cluster mean radial velocity (hereafter RV). The data and method of analysis are described in sections 2 and 3. Finally, a discussion of the statistical analysis and conclusions are given in sections 4 and 5.

\section{THE DATABASE}

A cluster had to fulfill the following selection criteria in order to be selected: (1) the clusters had to have Abell richness $(R)$ $\geq 1$, (2) the RV of the cluster should be known, and (3) the morphology of the cluster should be given. There were 107 clusters in the $\mathrm{ACO}^{7}$ catalogue fulfilling the selection criteria. We inspected all these clusters on the film copies (red sensitive ESO/POSS II) with the aid of a binocular microscope, and selected 34 of them. The reasons for the selection were: (1) the galaxies in these clusters were not too faint and it was relatively easier to determine diameters and position angles (hereafter PA) of many galaxies with relatively great accuracy, and (2) the Galactic contamination in and around the clusters was minimum, and (3) films were available. The search for galaxies was performed on the red sensitive $\operatorname{ESO}\left(\lambda_{\text {eff }}=640\right.$ $\mathrm{nm})$ and POSSII films $\left(\lambda_{\text {eff }}=640 \mathrm{~nm}\right)$ having a limiting magnitude of $m_{10}=20$.

Author for Correspondence: B. Aryal, Central department of Physics, Tribhuvan University, Kirtipur, Nepal. E-mail: binil.aryal@uibk.ac.at 


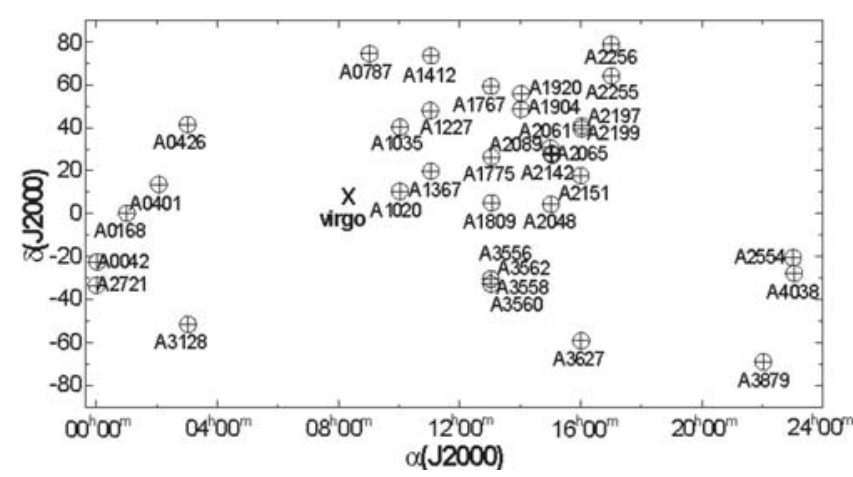

Figure 1: Distribution of 34 Abell clusters in the equatorial coordinate system. The Virgo cluster (symbol ' $\mathrm{X}$ ') can be seen.

Each ESO and POSSII film-copies of interest were scanned by eye. This was performed by the aid of a binocular microscope with a 25-fold magnification. The film-copies were illuminated from below. The criteria an object had to fulfill in order to be selected were: It had to show a diffuse, nonstellar appearance and major diameter $(a)$ of greater than 10 arcsec. The objects fulfilling these criteria were marked on transparent foils attached on the films. A list of all inspected film-copies is given in Table 1 . The second step was the re- examination of the objects selected in step 1 . Re-examination results the rejection of more than $20 \%$ of the objects. The remaining candidates were marked with a circle in the film for clear extragalactic identification.

A list of the investigated Abell clusters is given in Table 1. The data given in the second-sixth columns (cluster morphology, RV, the red magnitude of the tenth brightest cluster member $\left(m_{10}\right)$, richness $(R)$ and distance class $\left.(D)\right)$ in Table 1 are taken from the ACO catalogue. All sky distribution of the clusters is shown in Fig. 1. The database of well studied Virgo cluster is added ${ }^{8,9,10}$. The estimation of background galaxies in the cluster region was based on the area distribution of background galaxies around the cluster region. It is estimated that the background contamination of galaxies in the investigated cluster region is $\sim 10 \pm 5 \%$. We removed these galaxies from the database.

The foreground field galaxies in the investigated cluster region were identified with the help of the Uppsala General Catalogue of Galaxies ${ }^{9,10}$, the Third Reference Catalogue of Bright Galaxies ${ }^{11}$, the ESO/Uppsala Survey of the ESO (B) Atlas $^{12}$, Photometric Atlas of Northern Bright Galaxies ${ }^{13}$ and the Southern Galaxy Catalogue ${ }^{14}$. These field galaxies have been removed from the database.

Table 1: First column lists the Abell number. The next two columns give the type of print and the field. The positions of the cluster centre are listed in fourth and fifth columns. The sixth and seventh columns give the B-M type cluster morphology and the mean radial velocity of the cluster. The last two columns give the magnitude of tenth brightest cluster member and the number of galaxies in the cluster.

\begin{tabular}{|c|c|c|c|c|c|c|c|c|}
\hline Abell & Print & Field & R.A. (J2000) & Dec. (J2000) & B-M & RV (km/s) & $m_{10}$ & $\mathbf{N}$ \\
\hline 42 & ESO \$ & 473 & $00^{\mathrm{h}} 28^{\mathrm{m}} 36.5^{\mathrm{s}}$ & $-23^{\circ} 36^{\prime} 23^{\prime \prime}$ & I & 32587 & 17.1 & 165 \\
\hline 168 & POSSII & 825 & $00^{\mathrm{h}} 15^{\mathrm{m}} 09.8^{\mathrm{s}}$ & $+00^{\circ} 14^{\prime} 51^{\prime \prime}$ & II-III & 13466 & 15.4 & 175 \\
\hline 401 & POSSII & 616 & $02^{\mathrm{h}} 58^{\mathrm{m}} 56.9^{\mathrm{s}}$ & $+13^{\circ} 34^{\prime} 56^{\prime \prime}$ & I & 22424 & 15.6 & 211 \\
\hline 426 & POSSII & 301 & $03^{\mathrm{h}} 18^{\mathrm{m}} 36.4^{\mathrm{s}}$ & $+41^{\circ} 30^{\prime} 54^{\prime \prime}$ & II-III & 5486 & 12.5 & 166 \\
\hline 787 & POSSII & 37 & $09^{\mathrm{h}} 28^{\mathrm{m}} 33.7^{\mathrm{s}}$ & $+74^{\circ} 23^{\prime} 56^{\prime \prime}$ & II & 40532 & 16.9 & 98 \\
\hline 1020 & POSSII & 710 & $10^{\mathrm{h}} 27^{\mathrm{m}} 50.7^{\mathrm{s}}$ & $+10^{\circ} 24^{\prime} 40^{\prime \prime}$ & II-III & 19457 & 16 & 149 \\
\hline 1035 & POSSII & 317 & $10^{\mathrm{h}} 32^{\mathrm{m}} 07.3^{\mathrm{s}}$ & $+40^{\circ} 12^{\prime} 33^{\prime \prime}$ & II-III & 23953 & 15.4 & 157 \\
\hline 1227 & POSSII & 216 & $11^{\mathrm{h}} 31^{\mathrm{m}} 34.6^{\mathrm{s}}$ & $+48^{\circ} 01^{\prime} 33^{\prime \prime}$ & II-III & 33577 & 16.6 & 139 \\
\hline 1367 & POSSII & 572 & $11^{\mathrm{h}} 44^{\mathrm{m}} 29.5^{\mathrm{s}}$ & $+19^{\circ} 50^{\prime} 21^{\prime \prime}$ & II-III & 6595 & 13.5 & 166 \\
\hline 1412 & POSSII & 39 & $11^{\mathrm{h}} 55^{\mathrm{m}} 45.2^{\mathrm{s}}$ & $+73^{\circ} 28^{\prime} 18^{\prime \prime}$ & III & 25003 & 15.9 & 162 \\
\hline 1767 & POSSII & 132 & $13^{\mathrm{h}} 36^{\mathrm{m}} 00.3^{\mathrm{s}}$ & $+59^{\circ} 12^{\prime} 43^{\prime \prime}$ & II & 21045 & 15.7 & 158 \\
\hline 1775 & POSSII & 509 & $13^{\mathrm{h}} 41^{\mathrm{m}} 55.6^{\mathrm{s}}$ & $+26^{\circ} 21^{\prime} 53^{\prime \prime}$ & I & 20866 & 15.7 & 135 \\
\hline 1809 & POSSII & 793 & $13^{\mathrm{h}} 53^{\mathrm{m}} 18.9^{\mathrm{s}}$ & $+05^{\circ} 09^{\prime} 15^{\prime \prime}$ & II & 23624 & 15.8 & 128 \\
\hline 1904 & POSSII & 222 & $14^{\mathrm{h}} 22^{\mathrm{m}} 07.9^{\mathrm{s}}$ & $+48^{\circ} 33^{\prime} 22^{\prime \prime}$ & II-III & 21225 & 15.6 & 131 \\
\hline 1920 & POSSII & 175 & $14^{\mathrm{l}} 27^{\mathrm{m}} 16.9^{\mathrm{s}}$ & $+55^{\circ} 46^{\prime} 36^{\prime \prime}$ & II-III & 39273 & 17 & 167 \\
\hline 2048 & POSSII & 797 & $15^{\mathrm{h}} 15^{\mathrm{m}} 17.8^{\mathrm{s}}$ & $+04^{0} 22^{\prime} 56^{\prime \prime}$ & III & 28330 & 16 & 122 \\
\hline 2061 & POSSII & 449 & $15^{\mathrm{h}} 02^{\mathrm{m}} 15.3^{\mathrm{s}}$ & $+30^{\circ} 39^{\prime} 17^{\prime \prime}$ & III & 23024 & 15.7 & 129 \\
\hline 2065 & POSSII & 449 & $15^{\mathrm{h}} 22^{\mathrm{m}} 42.6^{\mathrm{s}}$ & $+27^{\circ} 43^{\prime} 21^{\prime \prime}$ & III & 21765 & 15.6 & 195 \\
\hline 2089 & POSSII & 450 & $15^{\mathrm{h}} 32^{\mathrm{m}} 41.3^{\mathrm{s}}$ & $+28^{\circ} 00^{\prime} 56^{\prime \prime}$ & II & 21945 & 15.8 & 111 \\
\hline 2142 & POSSII & 515 & $15^{\mathrm{h}} 58^{\mathrm{m}} 16.1^{\mathrm{s}}$ & $+27^{\circ} 13^{\prime} 29^{\prime \prime}$ & II & 26951 & 16 & 149 \\
\hline 2151 & POSSII & 584 & $16^{\mathrm{h}} 05^{\mathrm{m}} 15.0^{\mathrm{s}}$ & $+17^{\circ} 44^{\prime} 55^{\prime \prime}$ & III & 10943 & 13.8 & 190 \\
\hline 2197 & POSSII & 331 & $16^{\mathrm{h}} 28^{\mathrm{m}} 10.4^{\mathrm{s}}$ & $+40^{\circ} 54^{\prime} 26^{\prime \prime}$ & III & 9223 & 13.9 & 119 \\
\hline 2199 & POSSII & 331 & $16^{\mathrm{h}} 28^{\mathrm{m}} 37.0^{\mathrm{s}}$ & $+39^{\circ} 31^{\prime} 28^{\prime \prime}$ & $\mathrm{I}$ & 9156 & 13.9 & 117 \\
\hline 2255 & POSSII & 101 & $17^{\mathrm{h}} 12^{\mathrm{m}} 31.0^{\mathrm{s}}$ & $+64^{\circ} 05^{\prime} 33^{\prime \prime}$ & II-III & 24163 & 15.3 & 229 \\
\hline 2256 & POSSII & 23 & $17^{\mathrm{h}} 03^{\mathrm{m}} 43.5^{\mathrm{s}}$ & $+78^{\circ} 43^{\prime} 03^{\prime \prime}$ & II-III & 18018 & 15.3 & 162 \\
\hline 2554 & ESO \$ & 604 & $23^{\mathrm{h}} 12^{\mathrm{m}} 15.1^{\mathrm{s}}$ & $-21^{\circ} 33^{\prime} 56^{\prime \prime}$ & II & 31778 & 16.9 & 252 \\
\hline 2721 & ESO \$ & 349 & $00^{\mathrm{h}} 06^{\mathrm{m}} 14.5^{\mathrm{s}}$ & $-34^{\circ} 42^{\prime} 51^{\prime \prime}$ & II & 34386 & 17 & 274 \\
\hline 3128 & ESO \$ & 200 & $03^{\mathrm{h}} 30^{\mathrm{m}} 34.6^{\mathrm{s}}$ & $-52^{\circ} 33^{\prime} 12^{\prime \prime}$ & I-II & 17958 & 15.1 & 153 \\
\hline 3556 & ESO \$ & 444 & $13^{\mathrm{h}} 24^{\mathrm{m}} 06.2^{\mathrm{s}}$ & $-31^{\circ} 39^{\prime} 38^{\prime \prime}$ & I & 14360 & 16.4 & 163 \\
\hline 3558 & ESO \$ & 444 & $13^{\mathrm{h}} 27^{\mathrm{m}} 54.0^{\mathrm{s}}$ & $-31^{\circ} 29^{\prime} 00^{\prime \prime}$ & I & 14390 & 15.1 & 395 \\
\hline 3560 & ESO \$ & 383 & $13^{\mathrm{h}} 31^{\mathrm{m}} 50.5^{\mathrm{s}}$ & $-33^{\circ} 13^{\prime} 25^{\prime \prime}$ & $\mathrm{I}$ & 14660 & 15.1 & 159 \\
\hline 3562 & ESO \$ & 444 & $13^{\mathrm{h}} 33^{\mathrm{m}} 31.8^{\mathrm{s}}$ & $-31^{\circ} 40^{\prime} 23^{\prime \prime}$ & I & 16632 & 15.5 & 278 \\
\hline 3627 & ESO \$ & 137 & $16^{\mathrm{h}} 15^{\mathrm{m}} 32.8^{\mathrm{s}}$ & $-60^{\circ} 54^{\prime} 30^{\prime \prime}$ & I & 4881 & 14.2 & 65 \\
\hline 3879 & ESO \$ & 76 & $22^{\mathrm{h}} 27^{\mathrm{m}} 49.7^{\mathrm{s}}$ & $-69^{\circ} 01^{\prime} 41^{\prime \prime}$ & I-II & 20386 & 16.1 & 119 \\
\hline
\end{tabular}



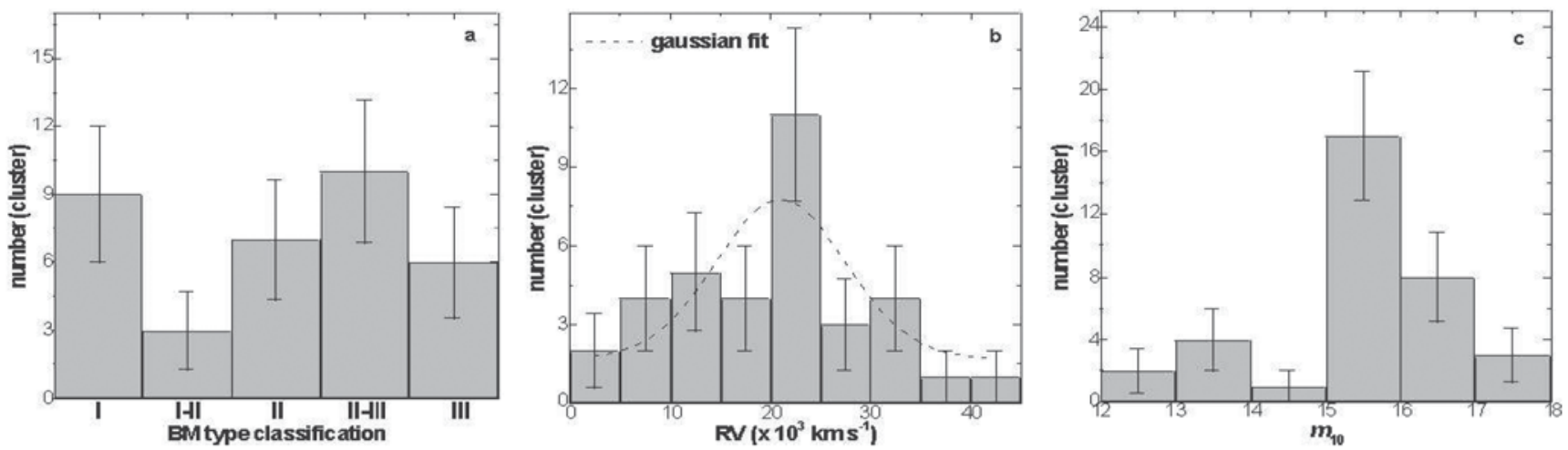

Figure 2: The distribution of BM (Bautz \& Morgan 1970) type classification (a), cluster mean radial velocity RV (b), and magnitude of the tenth brightest cluster member $\left(\mathrm{m}_{10}\right)(\mathrm{c})$. The observed values with statistical $1 \sigma$ error bars are shown.

Our database contains all types of clusters: regular or symmetrical (BM I type), intermediate (BM I-II, II, II-III) and irregular or asymmetrical (BM III type) (Fig. 2a). The maximum and minimum RVs of the clusters are 1,000 (Virgo) and 41,000 (A0787) km/s (Fig. 2b). There are 11 clusters that have mean RV 20,000 to 25,000 km/s in our database. Because of this bin, the center of the Gaussian fit is found at 21,000 km/s (Fig. 2b). There are 25 clusters that have $m_{10}$ value in the range 15 to 17 (Fig. 2c).

\section{METHODS: INCLINATION ANGLE}

Let us consider a disk inside a celestial sphere. Rotate the minor axis of the disk slowly without disturbing its major axis. Then, observe the projections in the celestial sphere. The observed image is the two dimensional projection on the celestial sphere. The axial ratios $(b / a)$ distributions of these projections obey cosine law. The cosine distribution remains intact when the rotational degree of freedom is increased. The disk galaxies are randomly distributed in the space.

The observed axial ratios of disk galaxies in the celestial sphere obey cosine distributions. Fig. 3a shows the expected axial ratios distribution curve. It is clear in the figure that the expected distributions of edge-on galaxies are less in number than the face-on. In the first three bins, $13.4 \%$ galaxies having $b / a<0.30$ are distributed (Fig. 3b). These are edge-on galaxies. In the next three bins $(0.3<b / a \leq 0.6)$ this number increases up to $36.6 \%$. In the last four bins $(0.6<b / a \leq 1.0), 50 \%$ galaxies are distributed. These galaxies are nearly face-on. The inclination angle ( $i$ ) is the angle between the normal to the galaxy plane and the observer's line-of-sight. The inclination angle can be computed from the formula ${ }^{16}$

$\cos ^{2} i=\frac{\left(q^{2}-q^{*^{2}}\right)}{\left(1-q^{*^{2}}\right)}$

This expression is valid for oblate spheroids. Here, $q$ and $q^{*}$ represent the measured axial ratio $(b / a)$ and the intrinsic flatness of the galaxy, respectively. The intrinsic flatness of a disk galaxy depends on the morphological type and is taken from Heidmann et al. (1971) ${ }^{15}$. Hiedmann et al. showed that the values of $q^{*}$ range from 0.083 for Sd spirals to 0.33 for ellipticals. For the galaxies with unknown morphology

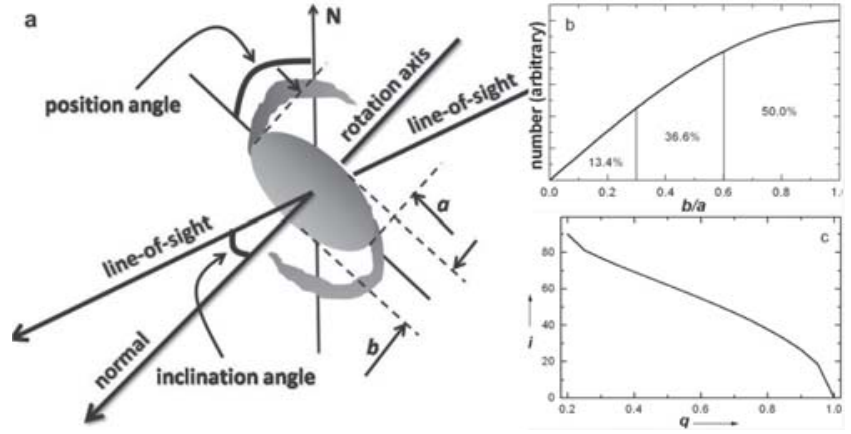

Figure 3: (a) Axial ratios distributions of disk galaxies in the celestial sphere. (b) The inclination angle (i) versus the axial ratios (q). The intrinsic flatness of the galaxy is assumed to be $0.20^{15}$.

$q^{*}=0.20$ is assumed. Fig 3 shows the plot between $q$ and $i$ for $q^{*}=0.20$.

It is clear from the Fig. 3 that the gradient of the inclination angle smoothly changes for the axial ratio range 0.25 to 0.95 . So, we can easily compute the inclination angle for the disk galaxies using Holmberg's formula given in equation (3). In order to remove the Holmberg effect we adopt Fouque \& Paturel method ${ }^{17}$ to convert measured diameters to standard photometric diameters. For this, we measured the diameters of 110 known galaxies (given in catalogue) and compared their diameters with the measured one. The ratio of the measured and given diameter is found to be 1.07 to 1.15 (7$15 \%$ more). We used an additive constant as suggested by FP for ESO and UGC catalogues in order to reduce visual diameters of galaxies to the photometric. We found that, our data needs an additive constant 0. '27 to the visual diameters to put them on a photometric system.

Astronomical data is often accompanied by several selection effects. One can regard these selection effects as a noise in the database. In such situations, even though all control parameters (independent variables) remain constant, the resultant outcomes (dependent variables) vary. A process of quantitatively estimating the trend of the outcomes, also known as regression or curve fitting, therefore becomes necessary. In this work, we have analysed the database of 5,700 galaxies in different context. For this we performed linear, polynomial and Gaussian fits in the inclination (i) angle 
distribution. The slopes of the fitted curves, the center and width of the Gaussian fits, the standard deviation of the fit and the correlation probability (that the correlation coefficient zero) are discussed.

\section{ANISOTROPY IN THE INCLINATION ANGLE DISTRIBUTION}

One major problem with applying the Holmberg model is the unique flatness parameter that is used for all types of galaxies. We used the flatness parameter as suggested by Haynes \& Giovanelli (1991) $)^{18}$ for morphologically identified galaxies
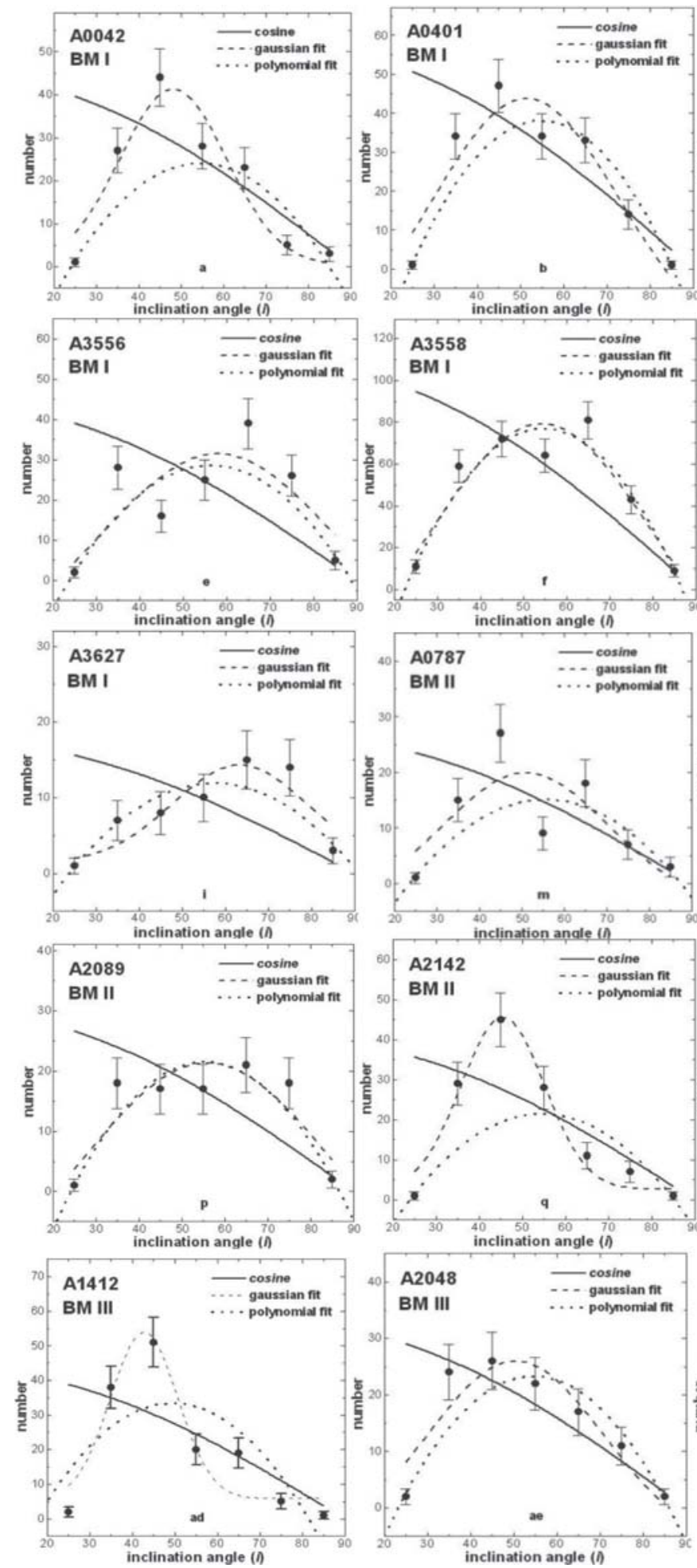

ranging from $q=0.23$ for ellipticals (E) and lenticulars (S0) to $q=0.10$ for late-type spirals (Scd, Sd). Morphologically unidentified galaxies dominate ( $\sim 60 \%$ ) our database. Many authors ${ }^{19,20,21}$ used $q=0.20$ for different galaxies. We used this value only for morphologically unidentified galaxies.

The observed image of the galaxy is the two dimensional projection on the celestial sphere. The axial ratios $(b / a)$ distributions of these projections obey cosine law. The inclination angle of face-on $(b / a=1)$ and edge-on $(b / a=0)$ galaxy is $0^{\circ}$ and $90^{\circ}$, respectively. Thus, $i$-distribution obeys cosine law. Fig. 4 shows the observed $i$-distribution of galaxies
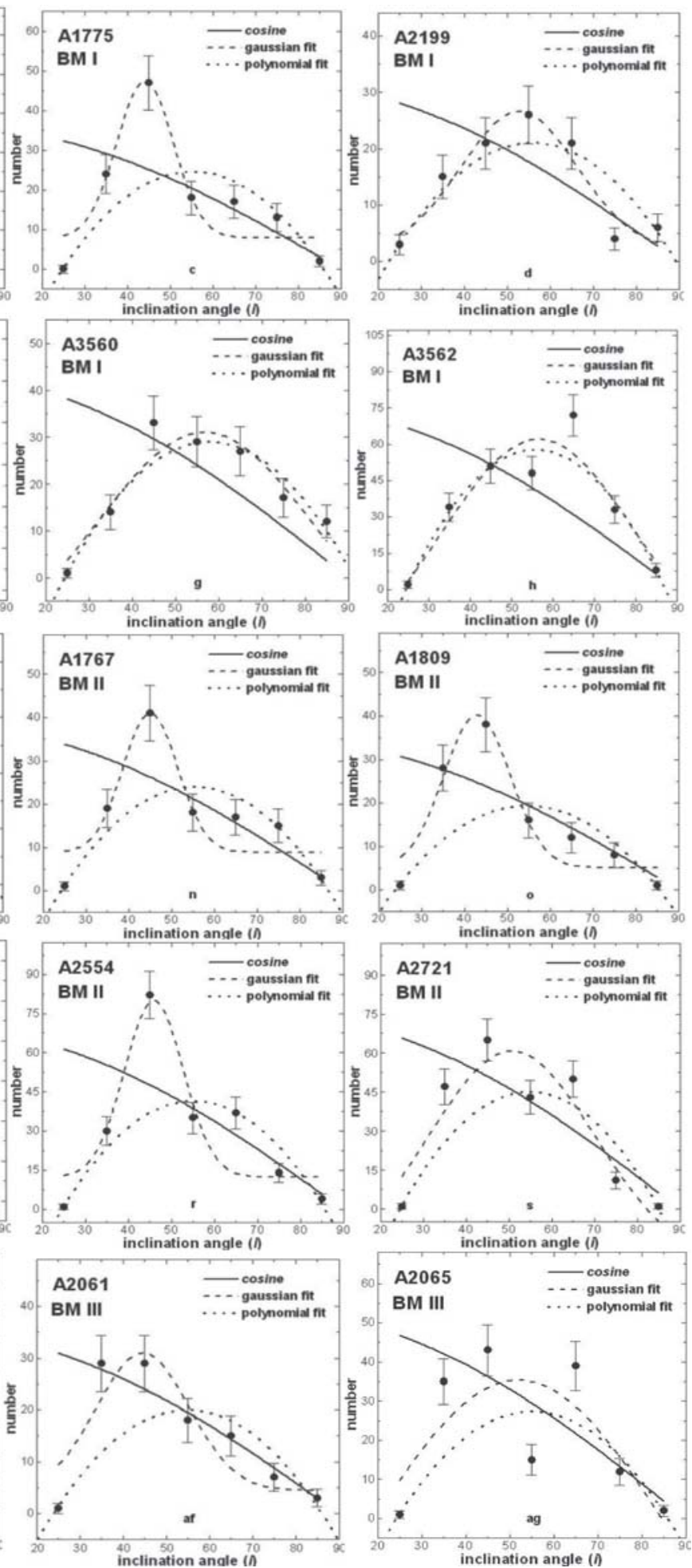

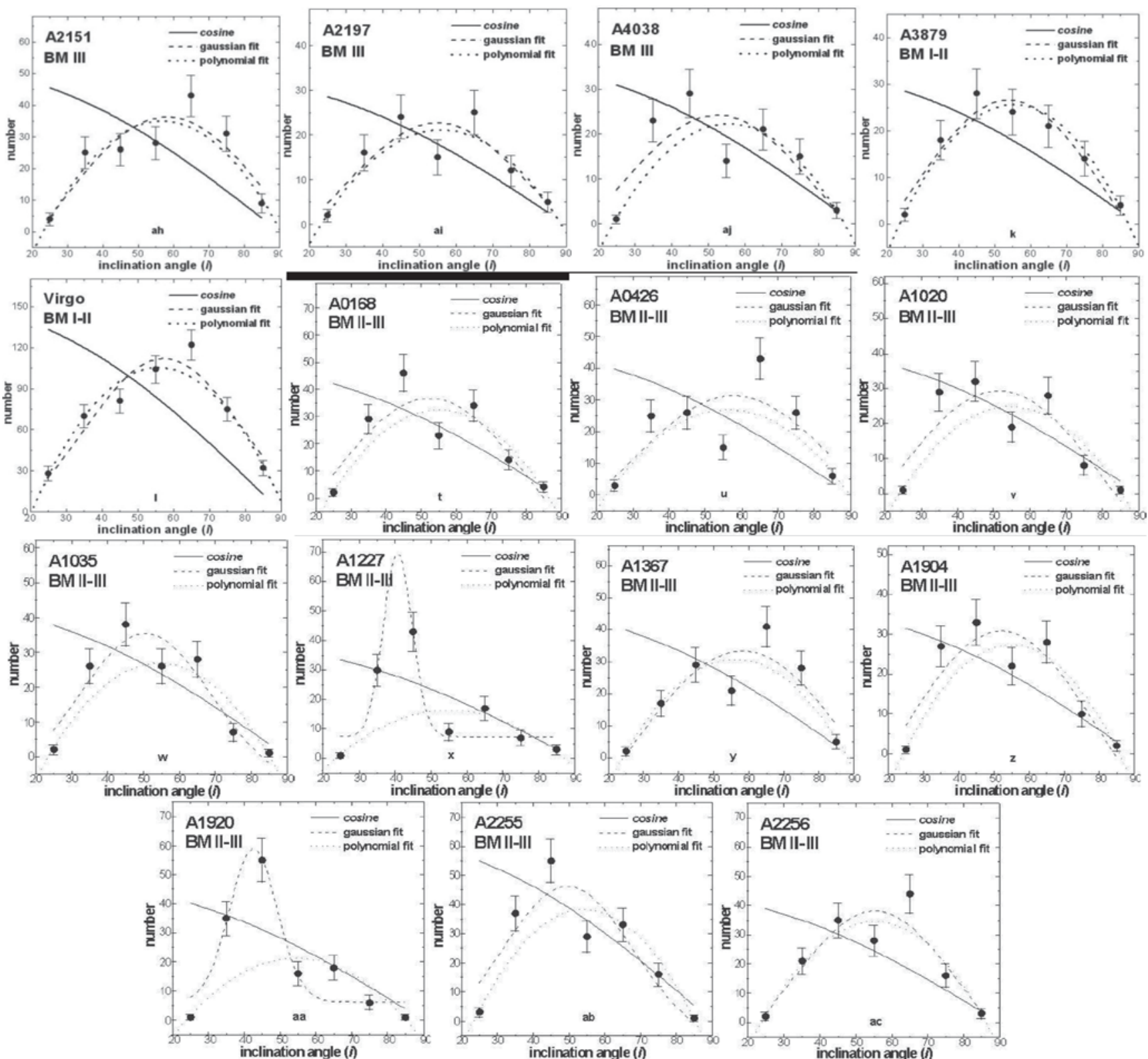

Figure 4: Inclination angle (i) distribution of galaxies in 35 clusters. The solid circles with statistical $\pm 1 \sigma$ error bars represent the observed idistribution. The Abell name and the BM type classifications are given. The solid curve represents the expected (cosine) distribution. The dashed and dotted curves represent the Gaussian and polynomial (second order) fits, respectively.

in 35 clusters. In the figure, solid curves represent the expected (cosine) distribution. The expected distribution of edge-on galaxies (low $i$ galaxies) are found to be less in number than the face-on. A large number of galaxies are missing in the first two (or three) bins $\left(i \leq 400^{\circ}\right)$ (Fig. 4). It is relatively difficult to measure PA of these missing (nearly face-on) galaxies accurately in the films. Few authors ${ }^{19,20}$ assumed $i=0^{\circ}$ for all missing nearly face-on galaxies. This may cause a heavy bias in $i$-distribution. In the middle of the histogram, the number of solutions is more than the expected (cosine) distribution. Thus, our database is dominated by nearly edge-on and edgeon galaxies.

In this work, we do not include galaxies that have $i<20^{\circ}$. In Fig. 4, the observed $i$-distribution is fitted using Gaussian (dashed curves) and second order polynomial (dotted curves). Fig. 4a shows $i$-distribution of galaxies in the cluster
A0042. The number of solutions in the first two bins $\left(i=25^{\circ}\right.$, $35^{\circ}$ ) are less in number than the expected. These are nearly face-on or face-on galaxies. In the third bin $\left(i=45^{\circ}\right)$, the galaxies are found to be more than the expected, suggesting a significant preference in $i$-distribution. The center of the Gaussian $\left(i^{\mathrm{GC}}\right.$ ) is found at $\sim 48^{\circ}$ (see Table 2).

In the Gaussian fit, the minimum and maximum values of the Gaussian center $\left({ }^{\mathrm{GC}}\right.$ in Table 2$)$ are found at $i=40.7^{\circ}$ (A1227, $\mathrm{D}=5$ ) and $62.3^{\circ}(\mathrm{A} 3627, \mathrm{D}=1)$, respectively. Similarly, the minimum and maximum values of the Gaussian width $\left(i^{\mathrm{GW}}\right.$ in Table 2$)$ are found at $\mathrm{i}=8.1^{\circ}(\mathrm{A} 1227, \mathrm{D}=5)$ and $70.0^{\circ}(\mathrm{A} 2065$, $\mathrm{D}=3$ ). The mean values of the $i^{\mathrm{GC}}$ and the $i^{\mathrm{GW}}$ are $51.4^{\circ}$ and $40.3^{\circ}$. Interestingly, the distant cluster $(D=5)$ showed minimum $i^{\mathrm{GC}}$ and $i^{\mathrm{GW}}$ values in $i$-distribution. In order to understand this, we have plotted $i^{\mathrm{GC}}$ of the cluster versus mean RV of the cluster (Fig. 5b). In the figure, a good agreement 
Table 2: The first column lists the Abell number. The next two columns give the values of the gaussian center (i $\mathrm{i}^{\mathrm{GC}}$ ), and the Gaussian width ( $\mathrm{i}^{\mathrm{GW}}$ ) in the i-distribution. The fourth and fifth columns represent the standard deviation ( $\mathrm{i}^{\mathrm{SD}}$ ) and the correlation probability $\left(\mathrm{i}^{\mathrm{P}}\right)$ in the polynomial fits of i-distribution. The next five columns repeat the first five columns.

\begin{tabular}{|c|c|c|c|c|c|c|c|c|c|}
\hline Abell & $i^{G C}$ & $i^{\mathrm{GW}}$ & $i^{\mathrm{SD}}$ & $i^{P}$ & Abell & $i^{G C}$ & $i^{\mathrm{GW}}$ & $i^{\mathrm{SD}}$ & $i^{P}$ \\
\hline 42 & 48.1 & 25.2 & 2.9 & 0.117 & 2721 & 50.7 & 38.8 & 3.1 & 0.041 \\
\hline 401 & 51.3 & 43.0 & 1.8 & 0.008 & 168 & 52.4 & 60.7 & 2.0 & 0.027 \\
\hline 1775 & 44.1 & 13.2 & 2.4 & 0.050 & 426 & 57.8 & 58.3 & 2.4 & 0.092 \\
\hline 2199 & 52.8 & 26.8 & 1.6 & 0.037 & 1020 & 51.4 & 63.6 & 2.1 & 0.035 \\
\hline 3556 & 57.9 & 53.5 & 2.0 & 0.037 & 1035 & 50.5 & 36.8 & 2.2 & 0.034 \\
\hline 3558 & 54.1 & 51.8 & 1.3 & 0.002 & 1227 & 40.7 & 8.1 & 3.2 & 0.250 \\
\hline 3560 & 56.8 & 50.3 & 1.0 & 0.002 & 1367 & 58.2 & 65.5 & 1.6 & 0.015 \\
\hline 3562 & 56.6 & 47.4 & 1.4 & 0.002 & 1904 & 52.0 & 56.3 & 1.8 & 0.017 \\
\hline 3627 & 63.3 & 31.1 & 1.0 & 0.018 & 1920 & 42.6 & 13.3 & 3.4 & 0.170 \\
\hline 3128 & 54.2 & 54.2 & 1.9 & 0.047 & 2255 & 49.6 & 36.7 & 2.1 & 0.015 \\
\hline 3879 & 53.8 & 56.3 & 0.7 & 0.001 & 2256 & 55.3 & 47.2 & 1.3 & 0.005 \\
\hline Virgo & 57.2 & 45.3 & 1.4 & 0.004 & 1412 & 42.8 & 15.8 & 15.0 & 0.179 \\
\hline 787 & 50.9 & 38.6 & 2.0 & 0.090 & 2048 & 50.4 & 41.2 & 1.3 & 0.010 \\
\hline 1767 & 45.1 & 12.9 & 1.9 & 0.030 & 2061 & 44.6 & 21.4 & 2.2 & 0.072 \\
\hline 1809 & 43.0 & 15.5 & 2.5 & 0.092 & 2065 & 51.9 & 70.0 & 3.0 & 0.090 \\
\hline 2089 & 55.9 & 58.0 & 1.1 & 0.005 & 2151 & 58.2 & 67.9 & 1.3 & 0.010 \\
\hline 2142 & 45.5 & 19.3 & 3.0 & 0.122 & 2197 & 54.9 & 52.9 & 1.2 & 0.015 \\
\hline 2554 & 46.0 & 13.7 & 3.0 & 0.045 & & & & & \\
\hline
\end{tabular}

between the linear and the polynomial fits can be seen. It is found that the $i^{\mathrm{GC}}$ value decreases with the increase of the mean RV of the cluster. We discuss this result later.

In the polynomial fit, the minimum and maximum values of the standard deviation $\left(i^{\mathrm{SD}}\right.$ in Table 3$)$ are $0.7(\mathrm{~A} 3879, \mathrm{D}=4)$ and 3.4 (A1920, D = 5), respectively (Fig. 4). A plot between the mean $\mathrm{RV}$ of the cluster and the $i^{\mathrm{SD}}$ value can be seen in Fig. 5b. The distant cluster (RV > 30,000 km/s) showed greater $i^{\mathrm{SD}}$ value. We linearly (solid line in Fig. 5b) and polynomially fitted (dashed curve in Fig. 5b) the observed distribution. No agreement between the linear and the polynomial fit can be seen. However, a weak dependence between the mean \$RV\$ of the cluster and the $i^{\mathrm{SD}}$ value cannot be denied. Higher the $i^{\mathrm{SD}}$ value, less reliable the preferred $i$. Thus, for the distant cluster, preferred $i$ value seem to be less reliable.

Twelve clusters showed the correlation probability $\left(i^{\mathrm{P}}\right)>5 \%$ (Table 2), suggesting a good agreement between the observed distribution and the polynomial fit. This value is found to be less than $0.1 \%$ for eight clusters. We do not notice significant dependence between the distance class and these statistical parameters.

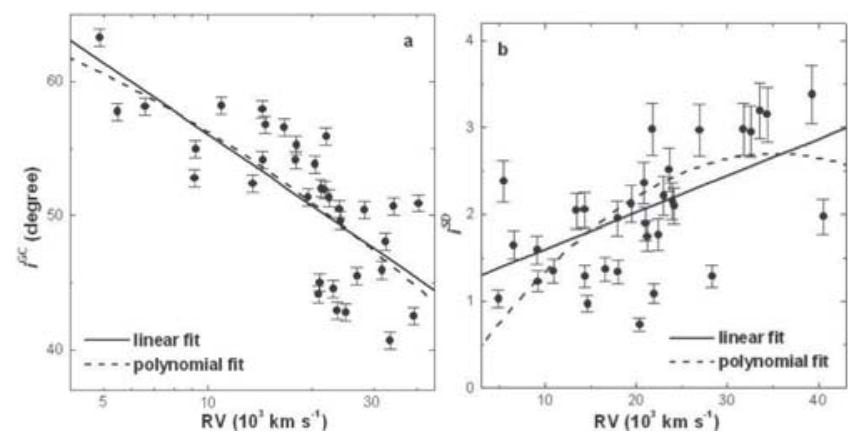

Figure 5: Gaussian center $\left(\mathrm{i}^{\mathrm{GC}}\right)$ of i-distribution versus cluster mean RV (b), and standard deviation of the polynomial fit in i-distribution $\left(i^{\mathrm{SD}}\right)$. A solid circle with error bars represents a cluster. The solid and dashed lines correspond to the linear and polynomial (second order) fits, respectively.

\section{DISCUSSION}

In $i$-distribution, the Gaussian center $\left(i^{\mathrm{GC}}\right)$ is found to be decreased with the increase of the mean RV of the cluster (Fig. 5b). The slope of the linear fit is found to be $-17.7 \pm 2.9$ with standard deviation $=5.9$. It seems that the distribution of preferred $i$ is not random in the cluster. The preferred \$i\$ depends upon the mean RV of the cluster. For distant cluster (high RV), the preferred value of $i$ is found to be less than that of the nearby cluster. This result might be due to the selection effects in the database. This effect should be taken into account in the three dimensional analysis. We noticed a weak dependence between the mean RV of the cluster and the standard deviation of the polynomial fit ( $i^{\mathrm{SD}}$ in Table 2, Fig. 5b) in $i$ distribution. For distant cluster, $i^{\mathrm{SD}}$ is found to be maximum, indicating less reliability. Obviously, the error in the measurement of diameters of the distant galaxies is high. In such case, the value of the preferred $i$ became less reliable. A weak relation is noticed between the preferred $i$ of the cluster and their mean RV. This dependence is less reliable for the distant cluster.

\section{CONCLUSION}

We measured diameters and the position angles of 5,688 galaxies in POSSII and ESO films using 25-fold magnification microscope. These galaxies belong to the 34 Abell cluster that have mean $\mathrm{RV} \sim 1,000$ to $43,000 \mathrm{~km} / \mathrm{s}$. The distribution of the major diameter, inclination and position angles of galaxies in the clusters is studied. In order to study the nature of the scatter plots in these distributions, we used linear, polynomial (second order) and Gaussian fits. The expected distributions are compared with the fitted curves.

We found a weak relation between the preferred inclination angles (i) and the mean RV of the cluster. For distant cluster, the preferred $i$ value is found to be less than that of the nearby cluster. These preferred alignments are found to be independent of the mean RV, distance and richness class of the cluster. 


\section{ACKNOWLEDGEMENTS}

We acknowledge Prof. R. Weinberger and Prof. Walter Saurer of Innsbruck University, Austria for insightful discussions. BA is thankful to Innsbruck University, Austria for providing financial assistance to visit Innsbruck University during JanMarch 2009. We acknowledge Mr. S. Paudel, Mr. R. Pokhrel and Mr. Sudeep Neupane for the help in the data reduction. This research has made use of the NASA/IPAC Extragalactic Database (NED) which is operated by the Jet Propulsion Laboratory, California Institute of Technology, under contract with the National Aeronautics and Space Administration.

\section{REFERENCES}

[1] Zeldovich, Ya.B. and Novikiv, I.D. 1975. Structure and Evolution of the Universe, Nauka, Moscow

[2] Peebles, P.J.E. 1988. Astrophysical Journal. 332: 17.

[3] Oort, J.H. 1970a. Science. 170: 1363.

[4] Fall, S.M. 1982. Progress in Cosmology. Proc. of the Oxford Intl. Symp., Oxford, Dordrecht, D. Reidel Publishing Co., 347-356.

[5] Peebles, P.J.E., 1969. Astrophysical Journal. 155: 393.

[6] Aryal, B. and Saurer, W. 2006. Monthly Notices Royal Astron. Soc. 366: 438.

[7] Abell, G.O., Corwin, H.G. and Olowin, R.P. 1989. Astrophysical Journal Supp. 70: 1
[8] Binggeli, B., Sandage, A. and Tammann, G.A. 1985: Astronom. Journal. 90: 1681.

[9] Nilson, P. 1973. Uppsala General Catalogue of Galaxies. Nova Acta Uppsala University, Ser. V:A, Vol.1.

[10] Nilson, P. 1974. Upps. Astron. Obs. Rep. 5.

[11] de Vaucouleurs, G., de Vaucouleurs, A., Corwin et al. 1991. Third Reference Catalogue of Bright Galaxies, Springer-Verlag, New York.

[12] Lauberts, A. 1982. ESO/Uppsala Survey of the ESO B Atlas. ESO, Garching bei Muenchen.

[13] Kodaira, K., Okamura, S. and Ichikawa, S. 1990. Photometric Atlas of Northern Bright Galaxies, Univ. of Tokyo Press, Tokyo.

[14] Corwin, H.G., de Vaucouleurs, A., de Vaucouleurs, G. 1985. Univ. Texas Monogr. Astron.4: 1.

[15] Heidmann J., Heidmann N., de Vaucouleurs G. 1972. Monthly Notice of Royal Astronomical Soc. 75:85.

[16] Holmberg, E. 1946. Medd. Lund. Astron. Obs. Ser VI, No. 117.

[17] Forque P. and Paturel G., 1985. Journal Astronomy \& Astrophysics.150:192.

[18] Haynes, M. and Giovanelli, R. 1984. Astronomical Journal. 89: 758.

[19] Godlowski, W. 1994. Monthly Notices Royal Astronomical Soc. 271:19.

[20] Hu, F.X., Wu, G.X., Su, H.J. and Liu, Y.Z. 1995. Journal Astronomy Astrophysics. 302:45.

[21] Aryal, B. and Saurer, W. 2005. Monthly Notices Royal Astronomical Soc. 360:125. 